\title{
Financiamento à ciência no Brasil: distribuição entre as grandes áreas do conhecimento
}

\author{
Daniella Borges Ribeiro ${ }^{1}$ \\ https://orcid.org/0000-0001-9264-7618 \\ Edineia Figueira dos Anjos Oliveira ${ }^{2}$ \\ https://orcid.org/ 0000-0002-4138-0842
}

\author{
Mirian Cátia Vieira Basílio Denadai ${ }^{3}$ \\ https://orcid.org/ 0000-0003-0713-9382
}

Maria Lúcia Teixeira Garcia ${ }^{4}$

https://orcid.org/ 0000-0003-2672-9310

\footnotetext{
${ }^{1}$ Universidade Federal de Viçosa, Departamento de Economia Doméstica, Curso de Serviço Social, Viçosa, MG, Brasil ${ }^{2}$ Universidade Federal do Espírito Santo, Programa de Pós-Graduação em Política Social, Vitória, ES, Brasil

${ }^{3}$ Universidade Federal do Espírito Santo, Departamento de Serviço Social, Vitória, ES, Brasil

${ }^{4}$ Universidade Federal do Espírito Santo, Departamento de Serviço Social, Programa de Pós-Graduação em Política Social, Vitória, ES, Brasil
}

Financiamento à ciência no Brasil: distribuição entre as grandes áreas do conhecimento

Resumo: Este artigo analisa a direção dada às pesquisas científicas aprovadas pelo CNPq entre os anos de 2011 e 2014, identificando a relação entre o público e o privado e a destinação de recursos financeiros para as grandes áreas do conhecimento. Trata-se de uma pesquisa documental baseada em dados coletados do sistema eletrônico do CNPq, de abordagem quali-quantitativa. Os dados demonstraram o progressivo direcionamento de recursos públicos para o financiamento de pesquisas em instituições privadas, bem como a desigual distribuição entre as áreas e grandes áreas de conhecimento

Palavras-chave: Ciência. CNPq. Financiamento.

\section{Financing for science in Brazil: distribution among major areas of knowledge}

Abstract: This article analyzes the direction given to scientific research approved by CNPq between the years 2011 and 2014 , identifying the relationship between the public and the private sector and the allocation of financial resources to large areas of knowledge. This is a documentary research based on data collected from the CNPq electronic system, with a qualitative quantitative approach. The data demonstrated the progressive targeting of public resources for the financing of research in private institutions, as well as the uneven distribution between areas and large areas of knowledge.

Keywords: Science. CNPq. Financing.

Recebido em 25.02.2020. Aprovado em 15.03.2020. Revisado em 24.06.2020.

(C) O(s) Autor(es). 2020 Acesso Aberto Esta obra está licenciada sob os termos da Licença Creative Commons

Atribuição-NãoComercial 4.0 Internacional (https://creativecommons.org/licenses/by-nc/4.0/deed.pt_BR), que permite copiar, distribuir e reproduzir em qualquer meio, bem como adaptar, transformar e criar a partir deste material, desde que para fins não comerciais e que você forneça o devido crédito aos autores e a fonte, insira um link para a Licença Creative Commons e indique se mudanças foram feitas. 


\section{Introdução}

Embora as pesquisas científicas no Brasil tenham sido desenvolvidas inicialmente em institutos próprios, há de se destacar que a expansão desta atividade se deu a partir da sua incorporação como uma atividade essencial nas universidades públicas (CURY, 2005). Essa assertiva se sustenta, pois cerca de $90 \%$ das pesquisas brasileiras são desenvolvidas nas universidades públicas, sendo o principal lócus desta produção os programas de pós-graduação stricto sensu (BUENO, 2014).

Se por um lado, o desenvolvimento da ciência é fundamental para descobertas importantes e que deveriam ser apropriadas por todos, por outro, o modo de produção capitalista anseia se apropriar dos resultados científicos em favor da reprodução ampliada ou alargada do capital ${ }^{1}$ (RIBEIRO, 2015). Assim, a produção de conhecimentos científicos pode auxiliar no enfrentamento das múltiplas expressões da questão social, mas pode também aprofundá-las, adensando as desigualdades sociais. Trata-se, portanto, de uma disputa entre classes sociais distintas em torno da produção e apropriação do conhecimento científico e de seus resultados.

Desde uma mirada histórica, vemos que a expansão

A EC 95/2016 “[...] desmonta

o Sistema Nacional de Ciência

e Tecnologia [...] o orçamento

do Ministério de Ciência,

Tecnologia e Inovação e

Comunicações para

investimento [...] está previsto

na ordem de um terço do que

era 10 anos atrás" [...] das pesquisas só foi possível a partir da incorporação desta atividade pela pós-graduação stricto sensu (CURY, 2005). Este nível de ensino ${ }^{2}$, que ainda era incipiente nas décadas de 1930 e 1940, contou com o apoio fundamental de duas instituições criadas em 1951 para a sua consolidação: o Conselho Nacional de Pesquisa (CNP) ${ }^{3}$ e a Campanha Nacional de Aperfeiçoamento de Pessoal de Nível Superior (CAPES). A criação destas instituições esteve ligada aos interesses da chamada modernização da universidade que neste momento a incluía como um importante espaço para a realização de pesquisas que possibilitassem o desenvolvimento tecnológico, entendido na época como prerrogativa para o desenvolvimento do país. Há também que se destacar que o desenvolvimento tecnológico aparecia como um elemento fundamental em período de guerra fria (CURY, 2005).

A Lei $\mathrm{n}^{\circ} 1.310$ de 15 de janeiro de 1951, sancionada pelo presidente Dutra, criou o Conselho Nacional de Pesquisas (CNP) como uma autarquia ligada a Presidência da República (BRASIL, 2015) ${ }^{4}$. Dada as divergências e a oposição dos Estados Unidos quanto a atividade nuclear, em 1956 esta atividade foi transferida para a Comissão Nacional de Energia Nuclear (CNEN) retirando do CNP esta atribuição. Não mais se envolvendo diretamente nas pesquisas nucleares para a fabricação da energia atômica o CNP passou a fomentar pesquisas nas áreas da física, biologia, tecnologia e ciências da terra. Somente em $1965^{5}$ as ciências humanas e sociais foram incluídas nas ações de fomento do CNP passando este órgão a estimular todas as áreas do conhecimento (OLIVEIRA, 2003). A Lei n ${ }^{\circ} 6.129$ de 6 de novembro de 1974 não só transformou o CNP em CNPq como o vinculou a Secretaria de Planejamento da Presidência da República. Esta Lei também instituiu o CNPq como fundação e colocou sob sua responsabilidade a elaboração dos Planos Básicos de Desenvolvimento Científico e Tecnológico (PBDCT) (BRASIL, 1974). Em 1985 quando foi criado o Ministério de Ciência e Tecnologia (Decreto ${ }^{\circ}$ 91.146/1985) o Conselho passou a fazer parte deste Ministério ${ }^{6}$ (BRASIL, 2015a).

Embora a realização de pesquisas ainda se dê majoritariamente nas universidades públicas, o Estado brasileiro fortaleceu o discurso da necessidade de alocação de recursos públicos para as instituições privadas e delegou às universidades públicas a captação de recursos no mercado, colocando em risco a autonomia dos pesquisadores frente às empresas financiadoras ${ }^{7}$. Dessa forma, o Estado brasileiro, ao invés de estimular ainda mais a ciência produzida nas universidades com dinheiro público e a sua socialização, permite o escoamento de recursos financeiros para a produção de pesquisas privadas e rentáveis ao capital. O cenário de disputa por recursos públicos para o desenvolvimento de pesquisas agrava-se ainda mais quando consideramos a aprovação da Proposta de Emenda à Constituição (PEC 95) em 2016, que impõe o congelamento dos gastos públicos pelos próximos 20 anos (BRASIL, 2016). A EC 95/2016 “[...] desmonta o Sistema Nacional de Ciência e Tecnologia [...] o orçamento do Ministério de Ciência, Tecnologia e Inovação e Comunicações para investimento [...] está previsto na ordem de um terço do que era 10 anos atrás" (SOCIEDADE...; 2018). Em termos concretos, em 2019, o Ministério da Educação teve congelados 5,8 bilhões e o Ministério da Ciência, Tecnologia e Inovação 2,1 bilhões (SAYURI, 2019).

É nesse cenário que é mister refletir sobre quais pesquisas estão sendo desenvolvidas e qual tem sido a direção dada às mesmas. Também é preciso saber se a produção de conhecimentos tem sido desenvolvida 
levando-se em consideração o princípio constitucional da autonomia científica. Este artigo tem como objetivo geral analisar a direção dada às pesquisas científicas aprovadas pelo CNPq entre os anos de 2011 e 2014, identificando a relação entre o público e o privado e a destinação de recursos financeiros para as grandes áreas do conhecimento. Sendo assim, este artigo discute o tema da ciência e a disputa em torno da sua produção e apropriação. Além disso, demonstra como dados retirados de sistemas eletrônicos podem ser usados para a produção de conhecimentos, para a realização de uma intervenção profissional qualificada e para a luta política, o que contempla os objetivos deste número da revista.

\section{Metodologia}

Trata-se de uma pesquisa documental, quali-quantitativa, com documentos de acesso público. Utilizamos os dados brutos encontrados no site do CNPq e dados encaminhados por esta agência via Sistema E-sic. Os dados qualitativos foram analisados por meio da análise de conteúdo e os quantitativos através da análise estatística descritiva. Para a coleta de dados inicialmente utilizamos o site do CNPq e por meio do link chamadas encerradas identificamos todos os editais lançados por esta agência entre os anos de 2011 e 2014. Em seguida, procedemos à leitura dos documentos, anotamos as características dos editais e excluímos do nosso estudo todos os editais de bolsas, Programa Ciência sem Fronteiras e auxílios para editoração e eventos. Foram elegíveis para esta pesquisa apenas os editais de apoio à pesquisa ${ }^{8}$ que receberam recursos que não fossem apenas bolsas.

Posteriormente, investigamos no site do CNPq (no link chamadas resultados) a quantidade de projetos que foram aprovados dos editais selecionados e consultamos uma tabela enviada pelo $\mathrm{CNPq}$ (por meio do sistema E-sic) a quantidade de projetos submetidos à esta agência, para sabermos qual a demanda versus quantidade de projetos aprovados. Para o levantamento dos recursos financeiros destinados às grandes áreas do conhecimento utilizamos o sistema Dados abertos ${ }^{9}$. Nesta fase de coleta de dados, notamos que os recursos financeiros distribuídos para as pesquisas por ano de aprovação do sistema Dados abertos, se comparado, não condiz com a quantidade de projetos aprovados da lista do site do CNPq do link chamadas encerradas. Isso ocorre, ou porque alguns pesquisadores recorrem, podendo alcançar a aprovação de seu projeto após o recurso, ou pelo fato dos recursos serem repassados em parcelas, podendo ser contabilizado o restante do montante no ano seguinte. Assim, para a sistematização dos valores recebidos para as pesquisas utilizamos as tabelas do sistema Dados abertos (porque possui informações sobre os valores repassados aos pesquisadores). Os valores financeiros foram deflacionados por meio da calculadora cidadã e o índice para a correção escolhido foi o IGP-DI. Separamos os valores repassados aos pesquisadores por ano e na calculadora cidadã utilizamos como data inicial dezembro do ano em que o recurso foi recebido e como data final dezembro de 2019.

Foram elegíveis para esta pesquisa 16 editais de apoio ao pesquisador lançados pelo CNPq no ano de 2011. A estes editais foram submetidos 18.716 projetos de pesquisa, sendo aprovados 4.801 projetos e encontrados na listagem de recursos financeiros, 4.701 projetos. Em relação ao ano de 2012 foram analisados 31 editais, para os quais houve 17.449 submissões, 5.278 projetos aprovados e 4.656 projetos encontrados na lista do sistema Dados abertos. No ano de 2013 foram escolhidos 55 editais, sendo encaminhados 30.732 projetos de pesquisa, dos quais 6.852 foram aprovados, e na listagem de recursos encontramos 8.415 pesquisas que receberam financiamento que não fossem apenas na modalidade bolsa. Quanto ao ano de 2014 foram analisados 28 editais, que receberam 23.445 submissões com 7.096 aprovações, e encontrados na lista de recursos 6.888 projetos. Desse modo, esta pesquisa conta com dados sobre 130 editais lançados pelo CNPq entre 2011 e 2014 cujos pesquisadores receberam recursos financeiros que não fossem apenas bolsas (Quadro 1).

\section{Quadro 1. Síntese dos Editais elegíveis para a pesquisa}

\begin{tabular}{c|c|c|c|c|c}
\hline Edital & $\begin{array}{c}\text { Número de Projetos } \\
\text { aprovados }\end{array}$ & $\begin{array}{c}\mathrm{N}^{\mathbf{o}} \text { de projetos encontrados com } \\
\text { os recursos financeiros recebidos }\end{array}$ & Edital & $\begin{array}{c}\text { Número de Projetos } \\
\text { aprovados }\end{array}$ & $\begin{array}{c}\mathrm{N}^{\mathbf{0}} \text { de projetos encontrados com } \\
\text { os recursos financeiros recebidos }\end{array}$ \\
\hline $01 / 2011$ & 12 & 12 & $30 / 2013$ & 13 & 13 \\
\hline $05 / 2011$ & 05 & 05 & $31 / 2013$ & 16 & 16 \\
\hline $06 / 2011$ & 200 & 200 & $33 / 2013$ & 13 & 13 \\
\hline $07 / 2011$ & 643 & 638 & $35 / 2013$ & 13 & 08 \\
\hline $08 / 2011$ & 08 & 08 & $36 / 2013$ & 09 & 12 \\
\hline
\end{tabular}




\begin{tabular}{|c|c|c|c|c|c|}
\hline $09 / 2011$ & 39 & 38 & $37 / 2013$ & 38 & Não encontrados \\
\hline $12 / 2011$ & 05 & 07 & $38 / 2013$ & 24 & Não encontrados \\
\hline $13 / 2011$ & 17 & 17 & $39 / 2013$ & 22 & Não encontrados \\
\hline $14 / 2011$ & 3.761 & 3.723 & $40 / 2013$ & 48 & Não encontrados \\
\hline $17 / 2011$ & 08 & 08 & $41 / 2013$ & 14 & Não encontrados \\
\hline $18 / 2011$ & 21 & 21 & $43 / 2013$ & 575 & 568 \\
\hline $19 / 2011$ & 58 & Não encontrados & $47 / 2013$ & 23 & 23 \\
\hline $20 / 2011$ & 08 & 08 & $48 / 2013$ & 26 & Não encontrados \\
\hline $21 / 2011$ & 09 & 09 & $49 / 2013$ & 24 & Não encontrados \\
\hline $22 / 2011$ & 04 & 04 & $51 / 2013$ & 15 & 15 \\
\hline $26 / 2011$ & 03 & 03 & $56 / 2013$ & 17 & Não encontrados \\
\hline $04 / 2012$ & 03 & 03 & $57 / 2013$ & 23 & 22 \\
\hline $10 / 2012$ & 29 & 29 & $62 / 2013$ & 07 & 07 \\
\hline $11 / 2012$ & 38 & 38 & $63 / 2013$ & 13 & 13 \\
\hline $13 / 2012$ & 04 & 04 & $64 / 2013$ & 20 & 19 \\
\hline $14 / 2012$ & 3.836 & 3.569 & $65 / 2013$ & 10 & 09 \\
\hline $15 / 2012$ & 09 & Não encontrados & $67 / 2013$ & 37 & 35 \\
\hline $16 / 2012$ & 24 & Não encontrados & $68 / 2013$ & 14 & 14 \\
\hline $17 / 2012$ & 265 & Não encontrados & $73 / 2013$ & 10 & 10 \\
\hline $18 / 2012$ & 452 & 449 & $75 / 2013$ & 22 & 22 \\
\hline $19 / 2012$ & 17 & 17 & $76 / 2013$ & 13 & 13 \\
\hline $20 / 2012$ & 03 & 03 & $77 / 2013$ & 12 & 12 \\
\hline $21 / 2012$ & 05 & Não encontrados & $79 / 2013$ & 91 & Não encontrados \\
\hline $23 / 2012$ & 17 & 17 & $80 / 2013$ & 66 & 55 \\
\hline $25 / 2012$ & 10 & 10 & $81 / 2013$ & 93 & Não encontrados \\
\hline $26 / 2012$ & 35 & 35 & $82 / 2013$ & 25 & Não encontrados \\
\hline $27 / 2012$ & 32 & 31 & $84 / 2013$ & 62 & Não encontrados \\
\hline $29 / 2012$ & 13 & 13 & $86 / 2013$ & 0 & 0 \\
\hline $30 / 2012$ & 08 & 08 & $88 / 2013$ & 06 & 06 \\
\hline $31 / 2012$ & 01 & Não encontrados & $89 / 2013$ & 86 & Não encontrados \\
\hline $32 / 2012$ & 145 & 141 & $91 / 2013$ & 65 & Não encontrados \\
\hline $34 / 2012$ & 26 & 26 & $94 / 2013$ & 318 & Não encontrados \\
\hline $35 / 2012$ & 43 & Não encontrados & $04 / 2014$ & 0 & 0 \\
\hline $36 / 2012$ & 29 & 30 & $05 / 2014$ & 17 & 17 \\
\hline $39 / 2012$ & 30 & 29 & $07 / 2014$ & 08 & 08 \\
\hline $40 / 2012$ & 76 & 77 & $11 / 2014$ & 89 & 87 \\
\hline $41 / 2012$ & 08 & 08 & $12 / 2014$ & 88 & Não encontrados \\
\hline $42 / 2012$ & 66 & 66 & $14 / 2014$ & 5.536 & 5.409 \\
\hline $43 / 2012$ & 04 & 04 & $16 / 2014$ & 0 & 103 \\
\hline $45 / 2012$ & 20 & 19 & $17 / 2014$ & 472 & 409 \\
\hline $46 / 2012$ & 22 & 22 & $18 / 2014$ & 03 & 03 \\
\hline $52 / 2012$ & 08 & 08 & $21 / 2014$ & 08 & 11 \\
\hline $05 / 2013$ & 12 & Não encontrados & $22 / 2014$ & 597 & 579 \\
\hline $06 / 2013$ & 24 & 20 & $26 / 2014$ & 16 & 16 \\
\hline $07 / 2013$ & 27 & 26 & $27 / 2014$ & 17 & 17 \\
\hline $08 / 2013$ & 31 & 30 & $28 / 2014$ & 16 & 16 \\
\hline $09 / 2013$ & 03 & 03 & $29 / 2014$ & 10 & 09 \\
\hline $12 / 2013$ & 05 & 05 & $30 / 2014$ & 19 & 19 \\
\hline $13 / 2013$ & 14 & 14 & $31 / 2014$ & 13 & 13 \\
\hline $14 / 2013$ & 4.629 & 7.250 & $32 / 2014$ & 14 & 14 \\
\hline $15 / 2013$ & 29 & 28 & $33 / 2014$ & 12 & 12 \\
\hline $16 / 2013$ & 06 & 01 & $34 / 2014$ & 14 & 14 \\
\hline $17 / 2013$ & 50 & Não encontrados & $35 / 2014$ & 15 & 15 \\
\hline $22 / 2013$ & 31 & 30 & $36 / 2014$ & 13 & 13 \\
\hline $23 / 2013$ & 28 & 26 & $37 / 2014$ & 14 & 14 \\
\hline $24 / 2013$ & 37 & 34 & $38 / 2014$ & 25 & 25 \\
\hline $26 / 2013$ & 06 & 06 & $39 / 2014$ & 19 & 19 \\
\hline $27 / 2013$ & 07 & 07 & $40 / 2014$ & 23 & 23 \\
\hline $28 / 2013$ & 18 & 18 & $46 / 2014$ & 15 & 15 \\
\hline $29 / 2013$ & 12 & 12 & $47 / 2014$ & 23 & 08 \\
\hline
\end{tabular}




\section{O CNPq e as demandas por recursos para pesquisas}

O número de projetos de pesquisas submetidos e aprovados pelo CNPq entre os anos de 2011 e 2014 demonstram que os editais funcionam não apenas para a indução de pesquisas por áreas e/ou temas considerados estratégicos, mas também para a otimização dos recursos disponíveis e a possível alocação de parte dos recursos para o setor privado, pois, alguns editais são específicos para o setor privado ${ }^{10}$, outros permitem a concorrência deste setor com as instituições públicas. Entre 2011 e 2014, dos 130 editais de apoio ao pesquisador analisados, foram submetidas 90.342 propostas das quais 24.660 foram aprovadas, ou seja, um atendimento de $27,3 \%$ da demanda. Os recursos, distribuídos por meio de editais, permitem duas constatações iniciais: menos de 1/3 das demandas é atendida; a distribuição dos recursos é desigual por áreas de conhecimento. Isso se agrava quando observamos os limites das universidades na alocação de recursos próprios para a realização de pesquisa.

Para a submissão de projetos de pesquisa ao CNPq existem alguns critérios previamente definidos nos editais. Dos 130 editais analisados, $108(83,1 \%)$ exigiam a titulação de doutor ao proponente responsável pelo projeto. Dessa forma, afirmamos que dos 24.660 projetos aprovados, pelo menos $22.900(92,9 \%)$ tiveram como proponentes pesquisadores doutores. Sobre as instituições que poderiam ser descritas como executoras dos projetos de pesquisa a serem financiados pelo CNPq, $04(3,1 \%)^{11}$ editais foram destinados exclusivamente às instituições públicas, os outros permitiram a participação do setor privado com fins lucrativos ou sem fins lucrativos.

É preciso salientar que o Estado tem incentivado o setor privado no que tange a pesquisa tanto repassando recursos financeiros, quanto permitindo a associação das universidades públicas às instituições privadas. Nesse sentido, podemos citar a Lei de Inovação Tecnológica (Lei no ${ }^{\circ}$ 0.973/2004) que possui três eixos principais: o estabelecimento de parcerias entre as IES e as empresas; o estímulo à participação das universidades e centros de pesquisa no processo de inovação e o incentivo à inovação tecnológica nas empresas (BRASIL, [2019]). Esta lei permite e incentiva a utilização das universidades públicas para a realização de pesquisas que serão apropriadas por empresas, dando um sentido extremamente utilitário a produção do conhecimento. ALei de Inovação Tecnológica se articula com a Lei do Bem (Lei no 11.196/2005) que permite a subvenção econômica e isenção fiscal às empresas que afirmarem, através da autodeclararão, o compromisso em realizar pesquisas voltadas à inovação tecnológica (LIMA, 2007). Por isso, alguns autores como Lima (2007) e Neves (2004) chamam a atenção para a conversão do professor universitário de instituição pública em empreendedor, sendo uma das leis que respaldam esta atividade a Lei de Inovação Tecnológica.

\section{Distribuição das propostas aprovadas por tema}

Dos 130 editais estudados, $121(93,1 \%)$ definiram os temas a serem estudados; $04(3,1 \%)$ definiram a área a ser pesquisada e apenas $05(3,8 \%)$ não definiram temas e áreas para as pesquisas. Os temas e áreas indicados estão listados nos quadros abaixo:

\section{Quadro 2. Temáticas induzidas em 2011.}

\begin{tabular}{c|l|c|c}
\hline $\begin{array}{c}\mathbf{N}^{\mathbf{0}} \mathbf{d o} \\
\text { edital }\end{array}$ & \multicolumn{1}{|c|}{ Temáticas } & $\begin{array}{c}\mathbf{N}^{\mathbf{0}} \text { de projetos } \\
\text { enviados }\end{array}$ & $\begin{array}{c}\text { Projetos encontrados com } \\
\text { os recursos financeiros }\end{array}$ \\
\hline 01 & $\begin{array}{l}\text { Energia e Meio ambiente, com foco em energias renováveis; Saúde e Meio ambi- } \\
\text { ente, com foco em doenças negligenciadas e alimentação e nutrição funcional. }\end{array}$ & 52 & 12 \\
\hline 05 & Matemática, Física e Biodiversidade. & 36 & 05 \\
\hline 06 & Sem tema e sem área previamente definidos ${ }^{12}$. & 565 & 200 \\
\hline 07 & Área de Ciências Humanas, Sociais e Sociais Aplicadas. & 1.559 & 638 \\
\hline 08 & Consolidação e melhoria da infraestrutura de parques tecnológicos. & 57 & 08 \\
\hline 09 & $\begin{array}{l}\text { Melhoria da infraestrutura de incubadoras para prestação de serviços a empresas } \\
\text { de base tecnológica. }\end{array}$ & 142 & 38 \\
\hline 12 & $\begin{array}{l}\text { Matemática; nanomagnetismo, estruturas e informação quântica; web Science; } \\
\text { computação de alto desempenho e gerenciamento de dados para aplicações de } \\
\text { alta performance; células-tronco e câncer; mudanças climáticas e desastres natu- } \\
\text { rais; doenças emergentes e mudanças ambientais. }\end{array}$ & 12 & 07 \\
\hline 13 & Bioma Caatinga. & 108 & 17 \\
\hline & & & \\
\hline
\end{tabular}




\begin{tabular}{|c|c|c|c|}
\hline 14 & Sem tema e sem área previamente definidos ${ }^{13}$. & 15.806 & 3.723 \\
\hline 17 & Nanociência e Nanotecnologia & 20 & 08 \\
\hline 18 & $\begin{array}{l}\text { Engenharias e demais áreas tecnológicas; Ciências Exatas e da Terra; Biologia, } \\
\text { Ciências Biomédicas e da Saúde; Computação e Tecnologias da Informação; } \\
\text { Tecnologia Aeroespacial; Fármacos; Produção Agrícola Sustentável; Petróleo, } \\
\text { Gás e Carvão Mineral; Energias Renováveis; Tecnologia Mineral; Tecnologia } \\
\text { Nuclear; Biotecnologia; Nanotecnologia e Novos Materiais; Tecnologias de Pre- } \\
\text { venção e Mitigação de Desastres Naturais; Tecnologias de transição para a eco- } \\
\text { nomia verde; Biodiversidade e Bioprospecção; Ciências do Mar; Indústria Cria- } \\
\text { tiva; Novas Tecnologias de Engenharia Construtiva; Formação de Tecnólogos. }\end{array}$ & 23 & 21 \\
\hline 19 & $\begin{array}{l}\text { Agricultura (carne, frutas e soja); Agronegócios; Agropecuária; Biodiversidade; } \\
\text { Biologia; Biomedicina; Biotecnologia, Ciência e Tecnologia de Alimentos; Ciência } \\
\text { e Tecnologia de Materiais; Ciência e Tecnologias Físicas; Ciências Agrárias; Ciên- } \\
\text { cias Aplicadas; Ciências Biomédicas; Ciências da Terra, Oceano e Atmosfera; } \\
\text { Ciências Espaciais; Ciências Físicas e Matemáticas; Ciências Humanas e Sociais; } \\
\text { Ciências Marinhas; Ciências Químicas; Competitividade Agroindustrial e Pes- } \\
\text { queira; Energia Nuclear e Espacial; Ensino de Ciências; Materiais Avançados; } \\
\text { Meio Ambiente; Microeletrônica, Mineração; Nanotecnologia; Produção e Sani- } \\
\text { dade Vegetal e Animal; Recursos Naturais; Recursos Renováveis e não-Renováveis; } \\
\text { Represas para Hidroelétricas; Risco e Vulnerabilidade Social para Eventos Natu- } \\
\text { rais e Ambientais; Saúde; Tecnologias da Informação e Comunicação; Tecnologias } \\
\text { Limpas; Todas as áreas do conhecimento; Turismo. }\end{array}$ & 269 & Não encontrado \\
\hline 20 & $\begin{array}{l}\text { Nanobiotecnologia (liberação controlada de fármacos e/ou nanoencapsulamento; } \\
\text { nanomateiriais para bioengenharia; nanopartículas para monitoramento e/ou di- } \\
\text { agnóstico em saúde; e, nanotoxicologia e/ou nanorregulação). }\end{array}$ & 18 & 08 \\
\hline 21 & $\begin{array}{l}\text { Nanotecnologia (Agronegócio, Eletrônica, Energia, Materiais, Meio ambiente, } \\
\text { Nanobiotecnologia, Saúde, Sensores e/ou reguladores e seus impactos). }\end{array}$ & 28 & 09 \\
\hline 22 & $\begin{array}{l}\text { Cartas de sensibilidade ambiental para derramamentos de óleo para quatro bacias } \\
\text { sedimentares marítimas: Bacia da Foz do Amazonas, Bacia do Pará-Maranhão/ } \\
\text { Barreirinhas, Bacia de Campos e Bacia de Pelotas. }\end{array}$ & 05 & 04 \\
\hline 26 & Segurança Alimentar, Bioenergia e/ou Biotecnologia Industrial. & 16 & 03 \\
\hline
\end{tabular}

Elaborado pelas autoras.

\section{Quadro 3. Temáticas induzidas em 2012.}

\begin{tabular}{c|l|c|c}
\hline $\begin{array}{c}\text { No do } \\
\text { edital }\end{array}$ & \multicolumn{1}{|c|}{ Temáticas } & $\begin{array}{c}\mathbf{N}^{\circ} \text { de projetos } \\
\text { enviados }\end{array}$ & $\begin{array}{c}\text { Projetos encontrados com } \\
\text { os recursos financeiros }\end{array}$ \\
\hline 04 & Nanotecnologia, materiais poliméricos & 05 & 03 \\
\hline 10 & Saúde bucal & 194 & 29 \\
\hline 11 & Urbanização & 1.011 & 38 \\
\hline 13 & Tecnologias da informação e comunicação & 61 & 04 \\
\hline 14 & Sem tema e sem área previamente definidos ${ }^{14}$. & 9.985 & 3.569 \\
\hline 15 & $\begin{array}{l}\text { Agricultura e alimentação, gestão de recursos naturais, mudanças climáticas, } \\
\text { desenvolvimento sustentável e humano, governança, tecnologia e inovação }\end{array}$ & 15 & Não encontrado \\
\hline 16 & Nanociência e nanotecnologia & 338 & Não encontrado \\
\hline 17 & Competitividade das empresas & 1.558 & Não encontrado \\
\hline 18 & Área Ciências humanas, sociais e sociais aplicadas. & 1.594 & 449 \\
\hline 19 & $\begin{array}{l}\text { Tecnologia da informação e comunicação, ciência e tecnologia para os oceanos, } \\
\text { ciências da vida, tecnologias limpas e energias renováveis }\end{array}$ & 36 & 17 \\
\hline
\end{tabular}




\begin{tabular}{|c|c|c|c|}
\hline 20 & Bioprodutos e bioprocessos aplicados à saúde humana & 109 & 03 \\
\hline 21 & Biotecnologia & 35 & Não encontrado \\
\hline 23 & Vigilância sanitária & 92 & 17 \\
\hline 25 & Métodos alternativos ao uso de animais e modelo de pele humana reconstituída & 48 & 10 \\
\hline 26 & Assentamento rural & 113 & 35 \\
\hline 27 & Inovação em educação alimentar e nutricional & 144 & 31 \\
\hline 29 & $\begin{array}{l}\text { Engenharias e demais áreas tecnológicas; Ciências Exatas e da Terra: Física, Química, } \\
\text { Geociências; Biologia, Ciências Biomédicas e da Saúde; Computação e Tecnologias da } \\
\text { Informação; Tecnologia Aeroespacial; Fármacos; Produção Agrícola Sustentável; Petró- } \\
\text { leo, Gás e Carvão Mineral; Energias Renováveis; Tecnologia Mineral; Tecnologia Nucle- } \\
\text { ar; Biotecnologia; Nanotecnologia e Novos Materiais; Tecnologias de Prevenção e Mitigação } \\
\text { de Desastres Naturais; Tecnologias de transição para a economia verde; Biodiversidade e } \\
\text { Bioprospecção; Ciências do Mar; Indústria Criativa; Novas Tecnologias de Engenharia } \\
\text { Construtiva; e Formação de Tecnólogos. }\end{array}$ & 19 & 13 \\
\hline 30 & Bioenergia e biomassa, energia solar, sistemas de distribuição de energia, tecnologia & 16 & 08 \\
\hline 31 & Financiamento em saúde & 16 & Não encontrado \\
\hline 32 & Gênero & 365 & 141 \\
\hline 34 & Ecologia & 107 & 26 \\
\hline 35 & Biodiversidade e ecossistemas & 122 & Não encontrado \\
\hline 36 & Terapia celular & 116 & 30 \\
\hline 39 & Arquipélago e ilhas oceânicas & 72 & 29 \\
\hline 40 & Doenças negligenciadas & 506 & 77 \\
\hline 41 & Doenças e biofarmácia & 34 & 08 \\
\hline 42 & Pesca e aquicultura & 410 & 66 \\
\hline 43 & Segurança alimentar, bioenergia, biotecnologia industrial. & 12 & 04 \\
\hline 45 & Biodiversidade brasileira & 210 & 19 \\
\hline 46 & Agroecologia e sistemas orgânicos de produção & 58 & 22 \\
\hline 52 & $\begin{array}{l}\text { Biotecnologia; Microeletrônica; Nanotecnologia; Pesquisa Aeroespacial; Energia Nucle- } \\
\text { ar; Ciências Ambientais; Ecologia; Ciências Agrárias; e Ciências da Saúde. }\end{array}$ & 48 & 08 \\
\hline
\end{tabular}

Elaborado pelas autoras.

\section{Quadro 4. Temáticas induzidas em 2013.}

\begin{tabular}{c|l|c|c}
\hline $\begin{array}{c}\mathbf{N}^{\mathbf{0}} \text { do } \\
\text { edital }\end{array}$ & \multicolumn{1}{|c|}{ Temáticas } & $\begin{array}{c}\text { No de projetos } \\
\text { enviados }\end{array}$ & $\begin{array}{c}\text { Projetos encontrados com } \\
\text { os recursos financeiros }\end{array}$ \\
\hline 05 & Saúde & 187 & Não encontrado \\
\hline 06 & Saúde & 164 & 20 \\
\hline 07 & Saúde & 138 & 26 \\
\hline 08 & Saúde & 91 & 30 \\
\hline 09 & $\begin{array}{l}\text { Alimentação funcional; Conversão de biomassa, microalgas e desagregação } \\
\text { microbiana; Biorremediação, biolixiviação, reabilitação ambiental e sensores } \\
\text { nanobiotecnológicos; Biofármacos; Biomateriais; e Biologia sintética. }\end{array}$ & 14 & 03 \\
\hline 12 & $\begin{array}{l}\text { Biotecnologia e saúde, especialmente produtos biotecnológicos; Agricultura, } \\
\text { incluindo bioenergia. }\end{array}$ & 678 & 05 \\
\hline 13 & $\begin{array}{l}\text { Tecnologias da Informação e Computação; Geociências, incluindo Oceano- } \\
\text { grafia e Mudanças Climáticas; Engenharia, Ciência dos Materiais e } \\
\text { Nanotecnologia; Ciências da saúde e biomédicas; Matemática; e Energias } \\
\text { renováveis, eficiência energética, e tecnologias de baixo carbono. }\end{array}$ & 66 & 14 \\
\hline
\end{tabular}




\begin{tabular}{|c|c|c|c|}
\hline 14 & Sem tema e sem área previamente definidos ${ }^{15}$. & 16.504 & 7.250 \\
\hline 15 & $\begin{array}{l}\text { Avaliação de medicamentos, produtos ou insumos nacionais prioritários para o Sistema Único } \\
\text { de Saúde (SUS) }\end{array}$ & 118 & 28 \\
\hline 16 & $\begin{array}{l}\text { HIV/AIDS; Malária; Tuberculose; Biotecnologia; Sistemas de Conhecimento Tradicional; Ener- } \\
\text { gia alternativa e renovável; Tecnologia da Informação e Comunicação. }\end{array}$ & 33 & 01 \\
\hline 17 & $\begin{array}{l}\text { Agricultura e Agronegócios; Alimentação e Nutrição; Aquicultura e Pesca; Arqueologia; Biociências } \\
\text { Moleculares; Biocombustíveis; Biodiversidade; Biotecnologia; Ciência e Tecnologia dos Mate- } \\
\text { riais; Ciências Aplicadas; Ciências Biomédicas; Ciências da Saúde; Ciências da Terra, Oceano e } \\
\text { Atmosfera; Ciências do Mar; Ciências Espaciais; Ciências Físicas; Ciências Humanas e Sociais; } \\
\text { Ciências Químicas; Computação e Telecomunicações; Controle de Qualidade; Desenvolvimen- } \\
\text { to Sustentável; Ecologia; Energia Nuclear; Energias Alternativas; Engenharias; Farmacologia de } \\
\text { Produtos Naturais; Geofísica do Petróleo; Geologia e Hidrologia; Gestão Tecnológica; Meio } \\
\text { Ambiente, Energia e Telecomunicações; Melhoramento Genético; Metalurgia e Mineração; } \\
\text { Metrologia; Mudanças Climáticas; Nanotecnologia e Novos Materiais; Oceanografia; Química; } \\
\text { Recursos Hídricos; Recursos Renováveis e não-Renováveis; Saúde; Tecnologia da Informação e } \\
\text { Comunicação; Tecnologias Limpas; Turismo e Artesanato.Obs. A Itália permitia a submissão de } \\
\text { projetos para todas as áreas do conhecimento. }\end{array}$ & 454 & Não encontrado \\
\hline 22 & $\begin{array}{l}\text { Aerodinâmica; Materiais de Aplicação Aeroespacial; Sistemas de Propulsão; Robótica e Armar } \\
\text { Inteligentes; Sensores de Uso Aeroespacial; Simuladores; Hipervelocidade; Estruturas; } \\
\text { Guiamento e Controle; Sistemas de Comando e Controles. }\end{array}$ & 288 & 30 \\
\hline 23 & Transporte aquaviário e da construção naval. & 104 & 26 \\
\hline 24 & Desenvolvimento social & 292 & 34 \\
\hline 26 & $\begin{array}{l}\text { Melhoramento de cultivares e de produtos da agropecuária, horticultura e aquicultura; } \\
\text { Biorremediação; Biorrefinarias e subsequente conversão de biomassa em energia e commodities } \\
\text { químicas; Desenvolvimento de enzimas com aplicação na indústria de alimentos; e Produtos e } \\
\text { ferramentas biotecnológicas com aplicação em saúde animal. }\end{array}$ & 37 & 06 \\
\hline 27 & $\begin{array}{l}\text { Desenvolvimento e inovação de bioprodutos e/ou bioprocessos aplicados à produção de vaci- } \\
\text { nas recombinantes para prevenção de zoonoses em animais. }\end{array}$ & 37 & 07 \\
\hline 28 & Engenharia de Sistemas Biológicos. & 191 & 18 \\
\hline 29 & $\begin{array}{l}\text { Desenvolvimento de novos produtos de alimentação, cosméticos, fármacos ou outras aplica- } \\
\text { ções industriais, relacionadas às seguintes espécies: Caju; Guaraná; Açaí e juçara; Umbu e } \\
\text { Jabuticaba. }\end{array}$ & 155 & 12 \\
\hline 30 & $\begin{array}{l}\text { Desenvolvimento de produtos e processos biotecnológicos (biolixiviação e biorremediação) } \\
\text { para minimizar ou solucionar problemas ambientais causados pela atividade humana, tais como } \\
\text { resíduos industriais, domésticos, agropecuários, provenientes de mineradoras, da produção de } \\
\text { petróleo e derivados, da produção de combustíveis, tratamento de esgoto e águas superficiais, } \\
\text { subterrâneas e residuais, da indústria pesqueira, poluentes, entre outros. }\end{array}$ & 185 & 13 \\
\hline 31 & Doenças Endócrinas e Metabólicas & 297 & 16 \\
\hline 33 & Tecnologia em Redes Elétricas Inteligentes & 78 & 13 \\
\hline 35 & Gerenciamento de Recursos Hídricos & 113 & 12 \\
\hline 36 & Conservação da água e manejo, recuperação e conservação do solo e da biodiversidade & 141 & 08 \\
\hline 37 & Mudanças Climáticas & 287 & Não encontrado \\
\hline 38 & Agronegócio (inovação tecnológica para insumos agrícolas) & 322 & Não encontrado \\
\hline 39 & Agronegócio (agregação de Valor às Cadeias Produtivas de Frutas e Lácteos). & 310 & Não encontrado \\
\hline 40 & Desenvolvimento e inovação voltados para a cadeia produtiva do biodiesel. & 397 & Não encontrado \\
\hline 41 & Política de saúde & 57 & Não encontrado \\
\hline 43 & Área Ciências Humanas, Sociais e Sociais Aplicadas & 1.492 & 568 \\
\hline 47 & Novas Terapias Portadoras de Futuro & 127 & 23 \\
\hline 48 & Agronegócio (produção agropecuária sustentável e agroecologia). & 131 & Não encontrado \\
\hline 49 & $\begin{array}{l}\text { Tecnologias para geração de energia solar fotovoltaica, LEDs e fontes luminosas inovadoras e } \\
\text { para sistemas de iluminação de alta eficiência }\end{array}$ & 129 & Não encontrado \\
\hline
\end{tabular}




\begin{tabular}{|c|c|c|c|}
\hline 51 & Inovação no setor mineral & 3.983 & 15 \\
\hline 56 & $\begin{array}{l}\text { Desenvolvimento e Inovação para a Produção de Biocombustíveis e Bioprodutos a partir de } \\
\text { Microalgas }\end{array}$ & 98 & Não encontrado \\
\hline 57 & Avaliação de Tecnologias em Saúde & 72 & 22 \\
\hline 62 & Pesquisa e Desenvolvimento em Ilhas Oceânicas & 56 & 07 \\
\hline 63 & Rede de Pesquisa em Biotecnologia Marinha & 58 & 13 \\
\hline 64 & Programa Antártico Brasileiro & 63 & 19 \\
\hline 65 & Pesquisa e Desenvolvimento em Meteorologia e Climatologia & 46 & 09 \\
\hline 67 & Coleções biológicas & 256 & 35 \\
\hline 68 & Atmosfera na Amazônia & 51 & 14 \\
\hline 73 & Medicamentos fitoterápicos oriundos de espécies nativas da flora brasileira & 276 & 10 \\
\hline 75 & $\begin{array}{l}\text { Desenvolvimento e Inovação em Tecnologia s de Conversão de Combustíveis com Foco em } \\
\text { Combustíveis Sólidos }\end{array}$ & 63 & 22 \\
\hline 76 & Desenvolvimento tecnológico em terras raras & 95 & 13 \\
\hline 77 & $\begin{array}{l}\text { Pesquisa, desenvolvimento e inovação em Biotecnologia na Amazônia Ocidental, com foco nas } \\
\text { áreas de fármacos, fitoterápicos e cosméticos. }\end{array}$ & 51 & 12 \\
\hline 79 & $\begin{array}{l}\text { Redes Regionais de Pesquisa em Biodiversidade e Biotecnologia, para o desenvolvimento sus- } \\
\text { tentável das Regiões Norte, Centro-Oeste e Nordeste, com vistas à conservação e ao uso } \\
\text { sustentável dos recursos naturais dos biomas abrangidos por essas regiões. }\end{array}$ & 247 & Não encontrado \\
\hline 80 & Economia Criativa & 168 & 55 \\
\hline 81 & Agroecologia e Sistemas Orgânicos de Produção & 171 & Não encontrado \\
\hline 82 & Segurança Alimentar e Nutricional & 67 & Não encontrado \\
\hline 84 & Tecnologia Assistiva & 134 & Não encontrado \\
\hline 86 & Censo dos trabalhadores de vigilância sanitária & 01 & 0 \\
\hline 88 & $\begin{array}{l}\text { Engenharias e demais áreas tecnológicas; Ciências Exatas e da Terra: Física, Química, Geociências; } \\
\text { Biologia, Ciências Biomédicas e da Saúde; Computação e Tecnologias da Informação; Tecnologia } \\
\text { Aeroespacial; Fármacos; Produção Agrícola Sustentável; Petróleo, Gás e Carvão Mineral; Ener- } \\
\text { gias Renováveis; Tecnologia Mineral; Tecnologia Nuclear; Biotecnologia; Nanotecnologia e } \\
\text { Novos Materiais; Tecnologias de Prevenção e Mitigação de Desastres Naturais; Tecnologias de } \\
\text { transição para a economia verde; Biodiversidade e Bioprospecção; Ciências do Mar;Indústria } \\
\text { Criativa; Novas Tecnologias de Engenharia Construtiva; e Formação de Tecnólogos. }\end{array}$ & 16 & 06 \\
\hline 89 & $\begin{array}{l}\text { Projetos de pesquisa, desenvolvimento tecnológico e extensão de incubadoras tecnológicas de } \\
\text { empreendimentos econômicos solidários }\end{array}$ & 108 & Não encontrado \\
\hline 91 & Desenvolvimento do Esporte em suas diferentes dimensões & 490 & Não encontrado \\
\hline 94 & $\begin{array}{l}\text { Aeroespacial e Defesa; Agropecuária; Alimentos; Automobilística; Biotecnologia; Calçados; } \\
\text { Construção Civil; Economia Criativa; Energia; Energia Nuclear; Energia Renovável; Fármacos e } \\
\text { Complexo Industrial da Saúde; Indústria Naval; Metal-mecânica; Mineração; Nanotecnologia; } \\
\text { Petróleo e gás; Pesca e Aquicultura; Tecnologia Assistiva; Tecnologias Educacionais; Tecnologias } \\
\text { da Informação e Comunicação (TICs); Tecnologias para Sustentabilidade; Têxtil/Vestuário. }\end{array}$ & 545 & Não encontrado \\
\hline
\end{tabular}

Elaborado pelas autoras.

Quadro 5. Temáticas induzidas em 2014.

\begin{tabular}{|c|c|c|c|}
\hline $\begin{array}{l}\mathrm{N}^{0} \text { do } \\
\text { edital }\end{array}$ & Temáticas & $\begin{array}{l}\mathrm{N}^{0} \text { de projetos } \\
\text { enviados }\end{array}$ & $\begin{array}{l}\text { Projetos encontrados com } \\
\text { os recursos financeiros }\end{array}$ \\
\hline 04 & Censo dos trabalhadores de vigilância sanitária & 03 & 0 \\
\hline 05 & Pesquisas em Vigilância Sanitária & 112 & 17 \\
\hline 07 & Biotecnologia & 29 & 08 \\
\hline
\end{tabular}




\begin{tabular}{|c|c|c|c|}
\hline 11 & Desenvolvimento territorial & 202 & 87 \\
\hline 12 & Ferramentas de software, hardware e/ou serviços de TI & 1.078 & Não encontrado \\
\hline 14 & Sem tema e sem área previamente definidos ${ }^{16}$ & 16.907 & 5.409 \\
\hline 16 & $\begin{array}{l}\text { Tecnologias ambientais e mitigação de mudanças climáticas; Biotecnologia e uso sustentável da } \\
\text { biodiversidade; Agricultura; Saúde e fármacos; Espaço, defesa e segurança nacional; Desenvol- } \\
\text { vimento urbano; Segurança pública; Fontes alternativas de energias renováveis, biocombustíveis } \\
\text { e bioenergia; Nanotecnologia; Pesquisa Nuclear; Tecnologia da informação e comunicação; Con- } \\
\text { trole e Gerenciamento de Tráfego Aéreo }\end{array}$ & 345 & 103 \\
\hline 17 & $\begin{array}{l}\text { Áreas temáticas estratégicas do Plano Brasil Maior e demais áreas: Aeroespacial e Defesa; } \\
\text { Agropecuária; Alimentos; Automobilística; Biotecnologia; Calçados; Construção Civil; Econo- } \\
\text { mia Criativa; Energia; Energia Nuclear; Energias Renováveis; Fármacos e Complexo Industrial } \\
\text { da Saúde; Gastronomia; Indústria Naval; Logística; Metalmecânica; Mineração; Nanotecnologia; } \\
\text { Petróleo e gás; Pesca e Aquicultura; Tecnologias Ambientais e para a sustentabilidade; Tecnologias } \\
\text { Assistivas; Tecnologias Educacionais; Tecnológicas de etnodesenvolvimento em terras indíge- } \\
\text { nas; Tecnologias da Informação e Comunicação (TIC); Têxtil/Vestuário; Tecnologias Sociais. }\end{array}$ & 1.536 & 409 \\
\hline 18 & Perdas pós-colheita de grãos & 15 & 03 \\
\hline 21 & Saúde da População Negra no Brasil & 89 & 11 \\
\hline 22 & Área Ciências Humanas, Sociais e Sociais Aplicadas & 1.260 & 579 \\
\hline 26 & Distúrbios Neuropsiquiátricos & 157 & 16 \\
\hline 27 & Doenças Neurodegenerativas & 218 & 17 \\
\hline 28 & Medicina Regenerativa & 68 & 16 \\
\hline 29 & Segurança Internacional e Defesa Nacional & 36 & 09 \\
\hline 30 & DST-Aids & 63 & 19 \\
\hline 31 & Doença de Chagas & 110 & 13 \\
\hline 32 & Leishmanioses & 132 & 14 \\
\hline 33 & Doenças Cardiovasculares & 151 & 12 \\
\hline 34 & Doenças Respiratórias Crônicas & 113 & 14 \\
\hline 35 & Doenças Raras & 39 & 15 \\
\hline 36 & Doenças Renais & 131 & 13 \\
\hline 37 & Helmintíases & 82 & 14 \\
\hline 38 & Sistemas Orgânicos de Produção de Base Agroecológica & 53 & 25 \\
\hline 39 & Agroecologia & 66 & 19 \\
\hline 40 & Sementes e Extrativismo & 85 & 23 \\
\hline 46 & $\begin{array}{l}\text { Segurança alimentar; Saúde pública; Desenvolvimento agrícola e pecuário; Inclusão social, e } \\
\text { Mudanças climáticas e eventos extremos. }\end{array}$ & 226 & 15 \\
\hline 47 & Saúde & 139 & 08 \\
\hline
\end{tabular}

Elaborado pelas autoras.

Em termos de frequência das palavras utilizadas nesses editais, destacamos as 10 mais recorrentes: Tecnologia(s) (49 vezes), Ciência(s) (41 vezes), Saúde (28), Biotecnologia (17), Desenvolvimento (16), Nanotecnologia (13), Energia (12), Materiais (10), Biodiversidade (9), Informação (9). É possível notar a tecnologia e inovação como centralidade nos editais, e a ciência sendo associada aos produtos configurando-se como uma ciência instrumental, como nos aponta Marilena Chauí (2001). Para esta autora, à universidade transformada em organização social temse solicitado um comportamento análogo ao funcionamento de empresas, inclusive com a encomenda de pesquisas para satisfazer as necessidades de determinados setores da economia. Como demonstramos, dos 130 editais pesquisados $121(93,1 \%)$ indicavam previamente o tema a ser pesquisado. Essa forte indução, limita as possibilidades de escolha dos pesquisadores quanto ao que e para quê pesquisar, o que nos permite afirmar que a autonomia científica da universidade e, consequentemente, dos pesquisadores nela inseridos está fortemente comprometida.

Esta direção dada à produção de conhecimentos no país vincula-se a um documento do Ministério da Ciência, Tecnologia, Inovações e Comunicações intitulado Livro Azul. Este livro foi publicado em 2010 (resultante das recomen- 
dações da $4^{\text {a }}$ Conferência Nacional de Ciência, Tecnologia e Inovação ocorrida no mesmo ano) e afirma a necessidade de transformação do conhecimento em inovação e na parceria entre as instituições de ensino, de pesquisa e as empresas, além da cooperação internacional. Esta parceria é enfatizada constantemente com elogios a Lei de Inovação e a Lei do Bem (BRASIL, 2010). O Livro Azul determina o incentivo de pesquisas cujos temas são relacionados à agricultura, bioenergia, tecnologias da informação e comunicação, saúde, exploração das reservas de petróleo e gás do pré-sal, tecnologia nuclear, espaço e defesa, tecnologias portadoras de futuro e outras energias ${ }^{12}$ (BRASIL, 2010).

\section{Recursos financeiros destinados às pesquisas}

Dos recursos repassados pelo CNPq referentes aos editais de 2011 observa-se que as ciências biológicas seguidas das ciências exatas e da terra receberam a maior parte dos recursos, $\mathrm{R} \$ 65.173 .468,12$ e $\mathrm{R} \$ 60.854 .645,11$ respectivamente. No ano de 2012 a grande área mais contemplada foi a ciências agrárias com o repasse de $\mathrm{R} \$$ 73.821.140,22. Em 2013 foram às engenharias $(\mathrm{R} \$$ 82.969.236,56), ciências da saúde $(\mathrm{R} \$ 81.462 .055,44) \mathrm{e}$ ciências biológicas $(\mathrm{R}$ \$ 81.013.293,94). Em 2014 as ciências da saúde (R \$ 119.970.805,86) e biológicas (R\$ 103.950.137,64) (Gráfico 1) (BRASIL, 2015). Observa-se que os menores investimentos em todos os anos pesquisados foram para a grande área de linguística, letras e artes; ciências humanas e ciências sociais aplicadas, corroborando com o debate realizado ao longo deste trabalho sobre a indução de pesquisas em outras áreas.

\section{Gráfico 1. Recursos para as pesquisas.}

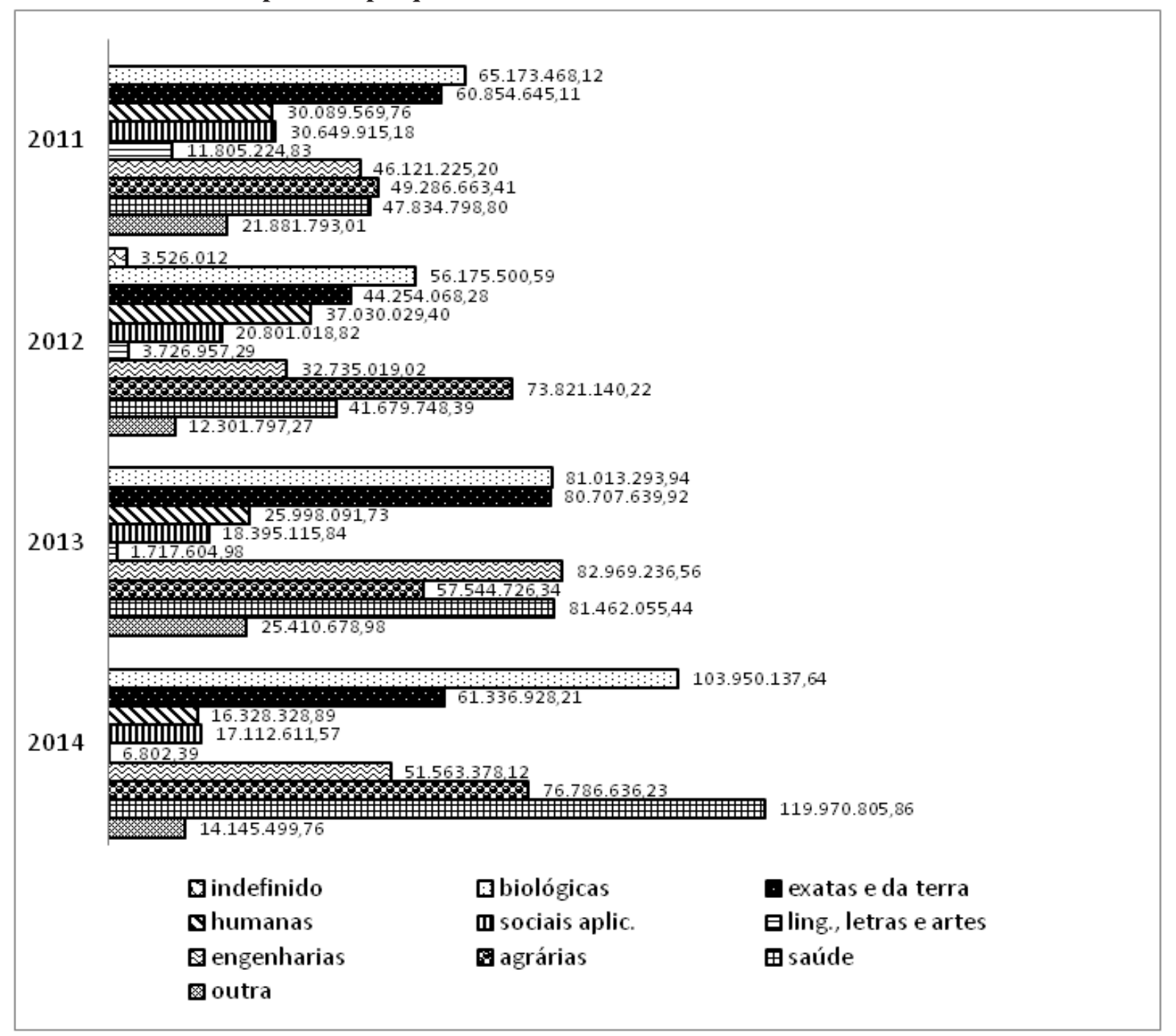

Elaborado pelas autoras, 2020. 


\section{Considerações Finais}

Essa pesquisa demonstra que o Estado cada vez mais se utiliza de estratégias legais para garantir o redirecionamento do fundo público para instituições privadas. $\mathrm{O}$ lançamento de editais para a realização de pesquisas com critérios mais flexíveis para a entrada de instituições privadas, sem a exigência de mestres e doutores, demonstra tais estratégias. As parcerias entre as instituições públicas e o setor privado são vistas como forma de incentivar as empresas frente à competitividade internacional. Como a realização de pesquisas demanda um alto financiamento, interessa ao empresariado que este investimento seja realizado com recursos do fundo público, mas que os seus resultados sejam apropriados pelo setor privado. Ou seja, embora as universidades ainda se constituam como os principais loci da produção de conhecimentos científicos, o Estado (através das agências de fomento) tem incentivado e financiado com dinheiro público pesquisas realizadas por empresas ou pesquisas realizadas por instituições públicas em parceria com as empresas.

Outra questão importante diz respeito à indução de pesquisas em determinadas áreas e temas, sendo linguística, letras e artes; ciências humanas e ciências sociais aplicadas as áreas que receberam o menor recurso para o desenvolvimento de pesquisas. Neste item é preciso considerar a prioridade dada para o desenvolvimento de pesquisas voltadas para o desenvolvimento do mercado, sustentando o processo de valorização do capital.

Embora não tenha sido aprovada uma única lei para regulamentar o princípio da autonomia universitária (e da autonomia científica) devemos considerar que a autonomia com o viés privatista já é normatizada por leis aparentemente isoladas (como a Lei de Inovação Tecnológica). Avaliamos assim, que o Estado e o mercado atuam no sentido de restringirem cada vez mais os relativos graus de autonomia científica das universidades públicas brasileiras.

Sendo assim, a produção de conhecimentos que poderia potencializar a satisfação das necessidades dos trabalhadores brasileiros encontra-se subordinada a criação de produtos e serviços a serem vendidos ou a processos que tem por objetivo aumentar a produtividade do trabalho. Há ainda de se mencionar que a venda dos resultados das pesquisas corrompem o compromisso científico do retorno coletivo dos resultados de estudos feitos com recursos públicos.

\section{Referências}

BARROS, E. M. C. de. Política de Pós-Graduação no Brasil (1975/1990): um estudo da participação da comunidade científica. São Carlos: EdUFSCar, 1998.

BRASIL. [Constituição (1988)]. Emenda constitucional no 95, de 15 de dezembro de 2016. Altera o Ato das Disposições Constitucionais Transitórias, para instituir o Novo Regime Fiscal, e dá outras providências. Brasília, DF: Presidência da República, 2016. Disponível em: http://www.planalto.gov.br/ccivil_03/constituicao/emendas/emc/emc95.htm. Acesso em: 17 fev. 2020.

BRASIL. Conselho Nacional de Desenvolvimento Científico e Tecnológico (CNPq). Investimentos do CNPq em CT\&I. Brasília, 2015. Disponível em: http://fomentonacional.cnpq.br/dmfomento/home/fmthome.jsp? Acesso em: 01 out. 2015.

BRASIL. Conselho Nacional de Desenvolvimento Científico e Tecnológico (CNPq). História do CNPq. Brasília, 2015a. Disponível em: http://centrodememoria.cnpq.br/Missao2.html. Acesso em: 17 jun. 2015.

BRASIL. Decreto $n^{\circ} 56.122$, de 27 de abril de 1965. Aprova o Regulamento do Conselho Nacional de Pesquisas. Brasília, DF: Presidência da República, 1965. Disponível em: https://www2.camara.leg.br/legin/fed/decret/1960-1969/decreto-56122-27-abril-1965396426-publicacaooriginal-1-pe.html. Acesso em: 17 fev. 2020.

BRASIL. Lei $n^{\circ}$ 10.973, de 2 de dezembro de 2004. Dispõe sobre incentivos à inovação e à pesquisa científica e tecnológica no ambiente produtivo e dá outras providências. Brasília, DF: Presidência da República, [2019]. Disponível em: http://www.planalto.gov.br/ ccivil_03/_ato2004-2006/2004/lei/110.973.htm. Acesso em: 17 fev. 2020.

BRASIL. Lei $n^{\circ} 6.129$ de 6 de novembro de 1974. Dispõe sobre a transformação do Conselho Nacional de Pesquisas em Conselho Nacional de Desenvolvimento Cientifico e Tecnológico (CNPq) e dá outras providências. Brasília, DF: Presidência da Republica, 1974. Disponível em: http://www.planalto.gov.br/ccivil_03/leis/1970-1979/L6129.htm. Acesso em: 17 fev. 2020.

BRASIL. Ministério da Ciência e Tecnologia. Centro de Gestão e Estudos Estratégicos. Livro Azul da $4^{a}$ Conferência Nacional de Ciência e Tecnologia e Inovação para o Desenvolvimento Sustentável. Brasília, 2010. Disponível em: http://www.mct.gov.br/upd_blob/ 0221/221783.pdf. Acesso em: 08 out. 2015.

BUENO, A. P. A Universidade e os projetos para o país. Revista Caros Amigos, São Paulo, ano 18, n. 70, p. 23-24, 2014.

CHAUÍ, M. Escritos sobre Universidade. São Paulo: Editora UNESP, 2001.

CURY, C. R. J. Quadragésimo Ano do Parecer CFE n. 977/65. Revista Brasileira de Educação, Rio de Janeiro, n. 30, p. 07-20, 2005. LIMA, K. R. de S. Contra-reforma na Educação Superior: de FHC a Lula. São Paulo: Xamã, 2007.

NEVES, L. M. W. A Reforma Universitária do governo Lula transforma a universidade em um negócio. Jornal da Associação dos Docentes da UFF, Niterói, p. 7 - 8, 1 ago. 2004. 
OLIVEIRA, A. de. Política Científica no Brasil: análise das políticas de fomento à pesquisa do CNPq. 2003. Dissertação (Mestrado em Educação) - Programa de Pós-Graduação em Educação, Universidade Federal de Santa Catarina, Florianópolis, 2003.

RIBEIRO, D. B. A Crise do Capital e seus Rebatimentos para a Produção de Conhecimentos. Revista Textos \& Contextos, Porto Alegre, v. 14, p. 314-326, 2015.

SAYURI, J. Os problemas da pós-graduação no Brasil e a importância da ciência. Nexo Jornal, 2019. Disponível em: https://www.nexojornal.com.br/ reportagem/2019/04/21/Os-problemas-da-pós-graduação-no-Brasil.-E-a-importância-da-ciência. Acesso em: 17 fev. 2020.

SOCIEDADE Brasileira de Progresso para a ciência pede fim da EC 95 a candidatos. SBPC na Mídia, 2018. Disponível em: http:// portal.sbpcnet.org.br/noticias/sociedade-brasileira-de-progresso-para-a-ciencia-pede-fim-da-ec-95-a-candidatos/. Acesso em: 17 fev. 2020.

\section{Notas}

1 Sugerimos o artigo de Ribeiro (2015) que discute as contribuições proporcionadas pela ciência moderna para o desenvolvimento das forças produtivas no capitalismo e como ela serve como meio de controle da força de trabalho e de valorização do capital.

2 Apesar da Lei de Diretrizes e Bases de 1961 (LDB) abordar a pós-graduação entendia-se que ela não era suficiente para a normatização do ensino nesse grau. Assim, o parecer CFEn ${ }^{\circ}$ 977/65, que foi homologado pelo Ministro da Educação em 1966, passou a conceituar e normatizar os cursos de pós-graduação no Brasil sendo este parecer um marco para a institucionalização da pós-graduação no país (CURY, 2005).

3 A Lei no 6.129 de 6 de novembro de 1974 transformou o Conselho Nacional de Pesquisas (CNP) em Conselho Nacional de Desenvolvimento Científico e Tecnológico(BARROS, 1998).

4 Este foi o primeiro órgão sistemático de coordenação da política de ciência e tecnologia (BARROS, 1998).

5 Decreto ${ }^{\circ}$ 56.122, de 27 de abril de 1965 (BRASIL, 1965).

6 Atualmente chamado de Ministério da Ciência, Tecnologia, Inovações e Comunicações (MCTIC).

7 Pensemos, por exemplo, na descoberta de vacinas e a venda destes produtos, o que deixará grande parte da população sem acesso a esse benefício.

$8 \mathrm{O} C N P q$ atua por meio do incentivo à capacitação de recursos humanos (assim como a CAPES) e também no fomento à pesquisa. O incentivo à capacitação é realizado mediante a concessão de bolsas de estudo no país e no exterior. Já o fomento à pesquisa acontece por meio do apoio aos projetos de pesquisa, a editoração e a realização de eventos (BRASIL, 2015a).

9 Endereço eletrônico: http://dadosabertos.cnpq.br/pt_BR/organization/cnpq.

10 Os editais 17/2012 e 12/2014, por exemplo, são específicos para empresas privadas com fins lucrativos.

11 Editais 94/2013; 17/2014; 40/2014; 11/2014.

12 Edital Casadinho/Procad.

13 Edital Universal.

14 Edital Universal.

15 Edital Universal.

16 Edital Universal.

17 Nas tecnologias portadoras de futuro "estão incluídas a nanotecnologia, a biotecnologia e algumas formas de energia que, embora já presentes em algumas aplicações, terão papel relevante na indústria do futuro. [...]Por conta da preocupação ambiental, outras formas de geração de energia, com baixa emissão de $\mathrm{CO} 2$, passarão a ser gradualmente implementadas. Entre as que terão participação crescente na matriz energética brasileira podem ser consideradas a energia fotovoltaica, a eólica, a utilização do hidrogênio nas células combustíveis e a energia nuclear" (BRASIL, 2010, p. 51).

\section{Daniella Borges Ribeiro}

dborgesribeiro@yahoo.com.br

Doutora pelo Programa de Pós-Graduação em Política Social da Universidade Federal do Espírito Santo (UFS)

Docente do curso de Serviço Social da Universidade Federal de Viçosa

\section{UFV}

Av. Peter Henry Rolfs, s/n, Campus Universitário

Viçosa - Minas Gerais - Brasil

CEP: 36570-900

\section{Edineia Figueira dos Anjos Oliveira}

eoliveiranjos@yahoo.com.br

Doutora pelo Programa de Pós-Graduação em Política Social da Universidade Federal do Espírito Santo (UFES) 
Mirian Cátia Vieira Basílio Denadai

mirianbasilio@yahoo.com.br

Doutora pelo Programa de Pós-Graduação em Serviço Social da Universidade Federal do Rio de Janeiro (UFRJ)

Docente do curso de Serviço Social da Universidade Federal do Espírito Santo (UFES)

\section{Maria Lúcia Teixeira Garcia}

lucia-garcia@uol.com.br

Doutora em Psicologia Social pela Universidade de São Paulo.

Docente do curso de Serviço Social e do Programa de Pós-Graduação em Política Social da Universidade Federal do Espírito Santo (UFES)

\section{UFES}

Av. Fernando Ferrari, 514, Goiabeiras

Vitória - Espirito Santo - Brasil

CEP: 29075-910

\section{Agradecimentos}

Aos (as) técnicos (as) do CNPq que nos auxiliaram respondendo as dúvidas enviadas pelo sistema E-Sic.

\section{Agência financiadora}

Coordenação de Aperfeiçoamento de Pessoal de Nível Superior [Edital CAPES/Print] número 88887.311889/2018-00, Fundação de Amparo à Pesquisa e Inovação do Espírito Santo [FAPES/ CAPES PROFIX 10/2018 e Edital FAPES/CNPq No 22/2018].

\section{Contribuições das autoras}

Daniella Borges Ribeiro - Coleta, Construção e redação do artigo. Edineia F. A. Oliveira - Redação, revisão e edição do trabalho.
Mirian Cátia V. B. Denadai - Redação, revisão e edição do trabalho.

Maria Lúcia T. Garcia - Redação, revisão e edição do trabalho.

Aprovação por Comitê de Ética e consentimento para participação

Não se aplica.

Consentimento para publicação

Consentimento das autoras.

Conflito de interesses

Não há conflito de interesses. 